\title{
Effect of Confinement Level and Local Heating on Healing Efficiency of Self-healing
}

\section{Particulate Composites}

\author{
Jonah Champagne ${ }^{1}$, Su-Seng Pang ${ }^{2}$, Guoqiang $\mathrm{Li}^{1, *}$ \\ ${ }^{1}$ Department of Mechanical \& Industrial Engineering, Louisiana State University, \\ Baton Rouge, LA 70803, USA \\ ${ }^{2}$ Macau University of Science and Technology, Avenida Wai Long, Taipa, Macau \\ *Corresponding author: Tel.: (225) 578-5302; Fax: (225) 578-5924; E-mail: lguoqi1 @ lsu.edu
}

\begin{abstract}
Shape memory polymer based self-healing materials are a special class of materials that have been the subject of much research over the past decade and many impactful discoveries have been made thus far. In a previous study (Compos. Sci. Technol. 2010; 70: 1419-1427) it was shown that in order for efficient healing, confinement during healing is essential. It was also shown that use of a two-step close-then-heal (CTH) method during healing of damaged shape memory particulate composites provided molecular scale healing. The present study further investigates the influence of confinement levels and local heating on the healing efficiencies of a polystyrene based shape memory polymer with $6 \%$ by volume of thermoplastic particle additives (copolyester as solid healing agent). The healing process was conducted by locally heating fractured cylindrical specimens at three levels of lateral (radial) confinements and axial constraints, and varying heating times. It is found that, lateral confinement levels, axial constraints, and heating times all control the healing efficiency. Almost complete strength recovery is obtained with $100 \%$ lateral confinement, $12 \mathrm{MPa}$ axial constraint stress, and 60 minutes of heating time.
\end{abstract}


Keywords: A. Polymer-matrix composites (PMCs); A. Smart materials; B. Damage tolerance; B. Mechanical properties; Self-healing;

\section{Introduction}

Crack formation and propagation in mechanical components has plagued the engineering and manufacturing industry for decades. As mechanical components are used, cracks may begin to form and propagate with continued use, and ultimately cause the component to fail, sometimes catastrophically. The problem is that many times cracks form without notice and through continued use of the component (cyclic loading) finally cause the component to fail. Damage due to impact is particularly problematic for fiber reinforced polymer composites because after the material has been impacted it may show no apparent damage on impact point and yet significantly damaged within. Self-healing materials offer a method of combatting this problem [1].

While a number of self-healing approaches and strategies have been explored for thermosetting polymers, and the literature is exploding, generally, healing can be divided into two categories. One is extrinsic healing based on incorporation of external healing agent; the other is intrinsic healing by polymer itself. As indicated by Li [2], both extrinsic healing and intrinsic healing can be further divided into physical healing (molecule entanglements) and chemical healing (reestablishment of chemical bonds). The chemical healing can be further divided into subcategories base on the type of chemical bonds (covalent bonds, hydrogen bonds, etc.).

Intrinsic healing systems include polymers with thermally reversible covalent bond (TRCB) [3], ionomer [4], supramolecule chemistry (hydrogen bonds, metal-ligand coordinations, $\pi-\pi$ stacking interactions) [5], thermosetting epoxy with unreacted epoxide [6], and dynamic 
covalent bond exchange (DCBE) [7]. However, not all the intrinsic self-healing polymers are suitable for applications in load carrying structures. The reason is that in load bearing structures, the polymer matrix must have sufficient strength and stiffness, even using fibers as reinforcement. Therefore, extrinsic healing systems by incorporation of external healing agent in thermosetting structural polymers have been developed . The healing agent can be liquid [8-13] or solid $[14,15]$. While the solid healing agent such as thermoplastic particles can be directly dispersed in a polymer matrix [16,17], liquid healing agent needs to be stored and released on demand through various containers such as microcapsules [18,19], hollow fibers [20-22], and biomimetic microvascular network $[23,24]$. This is a well-stablished research area, and is still growing very fast.

Based on Wool and O'Connor [25], for healing to occur, five stages of crack healing are needed, including (a) surface rearrangement; (b) surface approach; (c) wetting; (d) diffusion; and (e) randomization. For a lot of healing systems, the fractured specimens are usually manually brought into contact before healing starts. As indicated by Wool [26], and echoed by Binder [27] and $\mathrm{Li}$ [2], this simple operation represents the largest challenge in the real world applications. Obviously, one cannot bring a fractured skin panel together by hand in a Boeing aircraft. One may not be able to bring a fractured specimen together manually if the boundary of the specimen is fixed. If the specimen is forced to bring in contact, new damage may occur. Therefore, one grand challenge for both extrinsic healing and intrinsic healing is how to bring the fractured surfaces in contact before healing starts.

In 2008, Li and John [28] used shape memory effect to repeatedly close impact induced cracks in a sandwich composite. This idea was also extended to close impact induced cracks in grid stiffened composite [29] and 3-D woven fabric reinforced composite [30]. Later Li and 
Nettles [31] and Li and Uppu [32] proposed the two-step healing scheme, close-then-heal (CTH), by mimicking the self-healing mechanism of human skin; see Fig. 1. In this scheme, the shape memory polymer matrix was compression programed before service. Once a crack is created within the shape memory polymer matrix, heating triggers constrained shape recovery of the matrix, which helps close the crack, and melting, wetting, diffusion, and randomization of the embedded thermoplastic heal the narrowed or closed crack molecularly. It is noted that, in addition to closing cracks by compression programmed SMP matrix, crack closing can also be achieved by dual-functional polymeric system [33]. For conventional thermosetting polymers without shape memory effect, crack closing can be achieved by embedding pre-stretched SMP fibers [34], shape memory alloy (SMA) wires [35], and fishing line based polymeric artificial muscles [36]. It has been proved that this $\mathrm{CTH}$ scheme can heal structural scale cracks repeatedly, molecules, timely, and with a small amount of healing agent (as low as 3\% by volume) [16]. However, as indicated by $\mathrm{Li}$ and Uppu [32], a key requirement for CTH is that external constraint must be applied to effectively close the crack. And yet, it is not clear how the constraint level affects the healing efficiency. Also, in previous studies, the entire specimen or panel was heated to trigger constrained shape recovery, which is obviously impractical in real world structures. For example, one cannot heat the entire Boeing 787 in order to heal a crack at the tip of the wing.

The purpose of this study is to evaluate the effect of the level of external constraint and local heating time on the healing efficiency of a thermoset shape memory polymer based particulate composite, per the CTH scheme. 


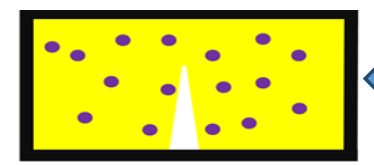

Damaged specimen confined 3deminsionally

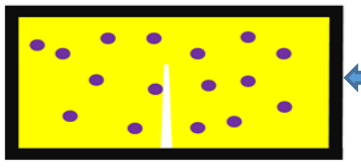

Heated to $T_{g}$ initiating SME of material and structurally closing crack

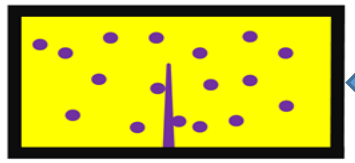

Heated to $T_{m}$ causing melting of $\mathrm{CP}$ and then to $T_{b}$ causing diffusion and bonding between CP and PSMP

Figure 1. Close-then-heal (CTH) scheme [2]. The golden color represents the polystyrene shape memory polymer (PSMP) and the purple dots represent the copolyester (CP) thermoplastic particles of the composite material.

\section{Experimentation}

\subsection{Raw Materials}

The material used in this study was a particulate composite. This material consisted of a polystyrene shape memory polymer (PSMP) matrix dispersed with copolyester thermoplastic particle additives (CP). Synthesis of the PSMP material was achieved by mixing vinylbenzene (Aldrich, Reagentplus $\geq 99 \%$ ), vinyl neodecanoate (Aldrich), divinyl-benzenene (Aldrich, technical grade, 80\%, mixture of ionomers) and polystyrene-block-polybutadieneblockpolystyrene (Sigma-Aldrich, Styrene, 30 Wt. \%). Benzoyl peroxide (Sigma-Aldrich, Luperox ${ }^{\circledR} \mathrm{A} 98$, reagent grade, $\geq 98 \%$ ) was used as the radical initiator. The thermoplastic particle additive was a linear copolyester (CP) consisting of Terepthalic acid, Isothalic acid, and 1,4-Butanediol (Abifor Inc., Switzerland). The copolyester possessed the following properties: particle size $\leq 80 \mu \mathrm{m}$, density $1.3 \mathrm{~g} / \mathrm{cm}^{3}$, glass transition temperature $T_{g} 16^{\circ} \mathrm{C}$, melting temperature $T_{m}$ range $114-124{ }^{\circ} \mathrm{C}$, and bonding temperature $T_{b}$ range $125-150{ }^{\circ} \mathrm{C}$. As a 
healing agent, the healing temperature needs to be in the range of $T_{b}$ in order to have sufficient physical entanglement between the healing agent molecules and the PSMP molecules.

\subsection{CP-PSMP composite fabrication and specimen preparation}

The fabrication of the CP-PSMP composite was achieved through a strategic mixing process followed by curing of the final material in an oven. To begin the process, vinylbenzene and polystyrene were added to a three-necked flask equipped with a mechanical stirrer. The mixture was then stirred for 30 minutes using a polytetrafluoroethylene stirrer. The remainder of the process was: divinyl-benzene was added and stirred for 30 minutes, vinyl neodecanoate was added and stirred for 30 minutes, $6 \%$ by volume of thermoplastic particles were added and stirred for 1 hour, and lastly benzoyl peroxide was added to the reactant to serve as the reaction initiator. The mixture was again stirred for 30 minutes. The mixture was then degassed in a Sargent-Welche vacuum (Welch brand, Duo-seal, Model NO. 1402 Ac-2065) at a 0.1-0.2 KPa vacuum for 30 minutes. After degassing, the mixture was poured into rectangular curing molds constructed from polytetrafluoroethylene. The top surface of the material was sealed using a polytetrafluoroethylene sheet. The material was cured as per the following scheme: 16 hours at $75^{\circ} \mathrm{C}, 10$ hours at $80^{\circ} \mathrm{C}$, and 5 hours at $110{ }^{\circ} \mathrm{C}$. After curing the composite was allowed to cool to room temperature after which it was demolded.

The cured rectangular slabs were cut into cylindrical specimens measuring $152 \mathrm{~mm}$ in length and having a $10 \mathrm{~mm}$ diameter. The shape and dimensions were chosen to comply with ISO178 standard for 3-point flexural tests. Also, the cylindrical shape removes any singularities within the specimen and provides uniform heat distribution during heating.

\subsection{Differential Scanning Calorimetry (DSC) analysis}


The $T_{g}$ of the CP-PSMP composite was determined via differential scanning calorimetry analysis. A $6.20 \mathrm{mg}$ sample of the CP-PSMP composite was placed in the sample pan in the Perkins Elmer DSC 4000 machine. The material was first held at $30{ }^{\circ} \mathrm{C}$ for one minute before it was cooled to $-70{ }^{\circ} \mathrm{C}$ at a rate of $20^{\circ} \mathrm{C} / \mathrm{min}$. The sample was then held for 30 minutes at $-70{ }^{\circ} \mathrm{C}$ afterwhich it was heated to $230{ }^{\circ} \mathrm{C}$ at a rate of $10{ }^{\circ} \mathrm{C} / \mathrm{min}$. It was then held at $230{ }^{\circ} \mathrm{C}$ for one minute before again being cooled to $-70{ }^{\circ} \mathrm{C}$ at a rate of $20^{\circ} \mathrm{C} / \mathrm{min}$. This heating and cooling cycle was performed two times and the curve produced from the second heating cycle was used for the analysis of the thermal properties of the CP-PSMP composite.

\subsection{Confinement tube preparation}

The confining tubes were constructed from a $0.2 \%$ carbon steel. Three tubes were fabricated to sizes that would provide 50,75 , and $100 \%$ radial confinement (i.e., 50, 75, and $100 \%$ of the length of the programmed specimens are within the confining tube). The outer diameter of each confining tube was $50.05 \mathrm{~mm}$ and inner diameter of $10.7 \mathrm{~mm}$. The cylindrical specimens had a length of $152.0 \mathrm{~mm}$ and diameter of $10.0 \mathrm{~mm}$. The lengths of the 50,75 , and $100 \%$ confining molds were 68.5, 102.7, and $137.0 \mathrm{~mm}$ respectively. Figure 2 illustrates a schematic of this setup. In using this method, 3-D confinement of the local damaged area can be realized as follows: the confining tube provides rigid lateral (radial) confinement in the local crack region, and the cold (un-heated and therefore rigid) part of the specimen lying outside the tube provides partial constraint in the axial direction by applying various external axial loads against free shape recovery. In other words, the axial compression force during healing represents the level of axial constraint so that, combined with the confinement by the tube, 3-D constraint is applied to the cylindrical specimen. Also, the heating zone (region 2 in Fig. 2) ensures that local heating can be conducted. 


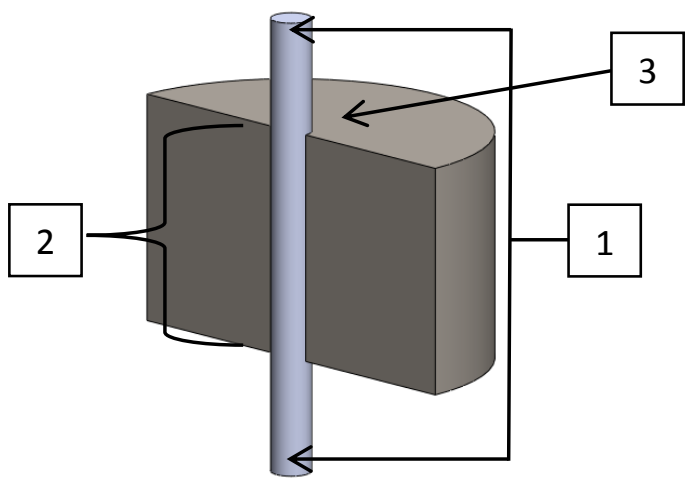

Figure 2. Local confinement setup: (1) Cold un-recovered region, (2) Local damaged area/region of interest, (3) Cutaway of partially confining cylindrical mold [14].

\subsection{Strain-controlled programming}

Each specimen underwent a strain-controlled compression programming to a $10 \%$ prestrain level. Strain controlled programming was chosen because it is much simpler to control the strain level during programming than that to control the stress. To perform the compression programming, the CP-PSMP cylindrical specimen was first wrapped in a small sheet of polytetrafluoroethylene and then placed inside the $100 \%$ confining tube (step one). This was done to facilitate easy removal of the specimen after the programming was complete. Next the specimen containing tube was placed inside a furnace that was coupled to a QTEST/150 MTS machine. The furnace was heated to $100{ }^{\circ} \mathrm{C}$ at a ramp rate of $6{ }^{\circ} \mathrm{C} / \mathrm{min}$ after which the specimen was given 1 hour to reach an equilibrium temperature (step 2). The specimen was then quasistatically strained at a rate of $1.3 \mathrm{~mm} / \mathrm{min}$ until it reached the designed compression strain level of $10 \%$. The furnace was cooled to room temperature while holding the programming strain constant, and the load was then removed (step 3). Figure 3 illustrates the programming process. 


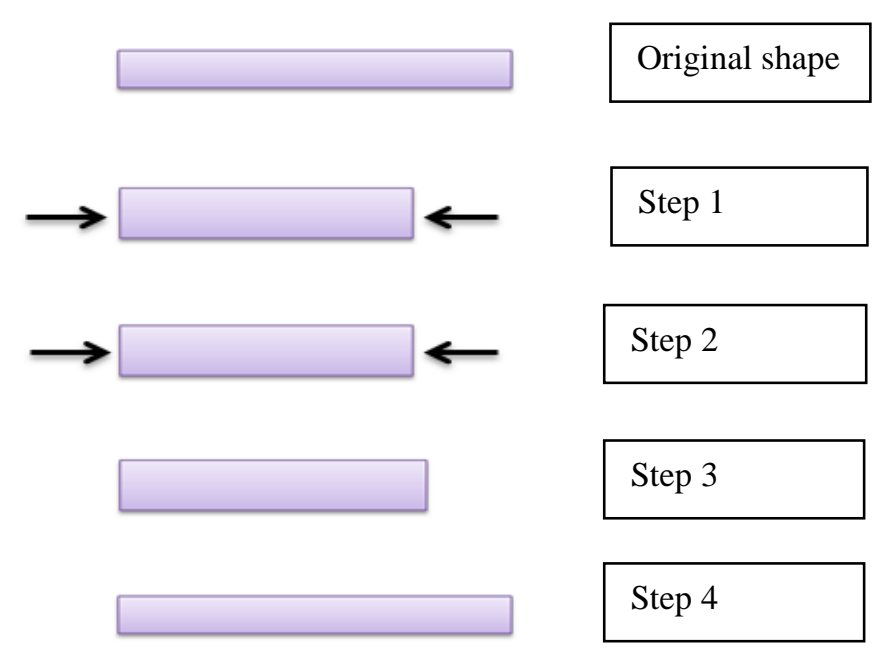

Figure 3. Thermomechanical cycle sequence. Step 1: CP-PSMP deformed at $T>T_{g}$. Step 2: CP-PSMP cooled to $T<T_{g}$ while constant strain is held. Step 3: Load removed, small springback occurs. Step 4: Shape recovery at $T>T_{g}$.

To complete the full thermomechanical cycle (discussed more in section 3.2) for a SMP based material, another step (step 4) is executed. In step 4 the SMP material is heated above its $T_{g}$ at which point the polymeric chains gain mobility and the shape memory effect ensues.

In this study multiple factors involved in step 4 are varied to evaluate their effect on the healing efficiency, namely, radial confinement level, axial constraint stress (healing stress), and healing time.

\subsection{Three-point flexural test}

Three-point flexural tests were performed as per ISO178 standards on un-notched specimens both before and after healing. The test was performed using a span of $86 \mathrm{~mm}$ which surpassed the minimal 8 to 1 span to diameter specifications for the ISO178 standard. The test was performed to complete specimen fracture at a rate of $0.5 \mathrm{~mm} / \mathrm{min}$. The strength regained after damage healing was compared to the original strength, which provided a means of quantifying the healing efficiency for the various testing procedures [37] via Eq. 1: 


$$
\eta=\frac{f_{\text {healed }}-f_{\text {damaged }}}{f_{\text {virgin }}-f_{\text {damaged }}}
$$

In Eq. $1, \eta$ is the healing efficiency and $f$ is the property being used to measure the efficiency against, in our case the flexural strength. The subscript "healed", "damaged", and "virgin" represent the strength after damage healing, after damage, and original undamaged specimens, respectively. In this study, the original specimen means the programmed specimen. Because the original specimen was completely fractured before healing, the term $f_{\text {damaged }}$ in Eq. 1 is zero.

\subsection{Radial (lateral) and axial constraints}

The main focus of this study was on the radial confinement level as well as the axial constraint stress. To that end, three levels of radial or lateral confinement (50, 75, and 100\%) and three levels of applied axial stress $(0,7$, and $12 \mathrm{MPa})$ were investigated. At this point, it is necessary to provide a formal definition of what each of these parameters physically mean. Firstly, the confinement level $(50,75$, and $100 \%)$ refers to the percentage of the major length of the specimen radially confined during healing by a rigid confining tube (as shown in Figure 2). The applied axial stress $(0,7$, and $12 \mathrm{MPa})$ refers to the amount of pressure applied to the ends of the specimen in the axial direction against free shape recovery it the axial direction. Figure 4 shows a flowchart of the experimental process followed in this study. In conjunction with the

partially confining tubes, XtremeFLEX BSO Silicone Rubber Heating tape (Briskheat Inc, Columbus Ohio) was used to provide localized heating to the confined area of the specimen. This was done by wrapping the tape around the confining tube as per the manufacturer's specifications and using a temperature controller to control the heating process.

\subsection{Healing time}


In addition to the confinement levels, the effect of varying the healing time was also investigated in this study. To that end, the applied axial stress and lateral confinement level were held constant at $12 \mathrm{MPa}$ and $100 \%$ respectively during this portion of the study. After undergoing 3-point flexural tests to complete failure, the specimens were placed in the $100 \%$ confining tube and heated to the healing temperature of $135^{\circ} \mathrm{C}$ at a ramp rate of $6{ }^{\circ} \mathrm{C} / \mathrm{min}$. Once the confining tube reached the healing temperature, an additional 30 minutes was allowed for the specimen within the tube to reach this equilibrium temperature. Next, the specimens were allowed to heal for additional 5, 30, or 60 minutes. Three-point flexural test was performed on the healed specimen in order to quantify the healing efficiency via Eq. 1.

\subsection{SEM observation}

A Hitachi S-3600N scanning electron microscope was utilized in order to study the crack interface of specimens healed under varying conditions. Images of the crack surface of specimens healed under 50\% confinement with $0 \mathrm{MPa}$ axial stress and specimens healed under $100 \%$ confinement and $7 \mathrm{MPa}$ axial stress were captured.

\section{Results and Discussions}

\subsection{Differential scanning calorimetry (DSC) results}

Differential Scanning Calorimetry (DSC) was employed to determine the $T_{g}$ of the CPPSMP composite material. Knowledge of the materials $T_{g}$ was imperative in order to know what temperatures could be used for programming purposes. The linear copolymer's $T_{g}$ value supplied by the manufacture was $16^{\circ} \mathrm{C}$ and the $T_{g}$ of the pure PSMP was determined by Yougoubare and Pang to be $\sim 89^{\circ} \mathrm{C}[38]$. Analysis of the curve produced by the DSC (shown in Figure 5) suggests two values for the $T_{g}$ of the material; one at $12.74{ }^{\circ} \mathrm{C}$ and one at $85.33{ }^{\circ} \mathrm{C}$. Because the material primarily consists of the PSMP (94\% by volume), the $T_{g}$ of the composite is mostly influenced 
by the $T_{g}$ of the pure PSMP. Therefore, it is expected that the $T_{g}$ of the composite be closer to the $T_{g}$ of the pure PSMP. This is indeed the case as can be seen in Figure 5. The composite material shows two values for the $T_{g}$, however, the smaller value is likely due to the presence of the CP which has a value close to the first one in the figure. Therefore, the larger value of $85.33^{\circ} \mathrm{C}$ was taken as the value of the $T_{g}$ for the CP-PSMP material and the programming temperature was chosen accordingly.

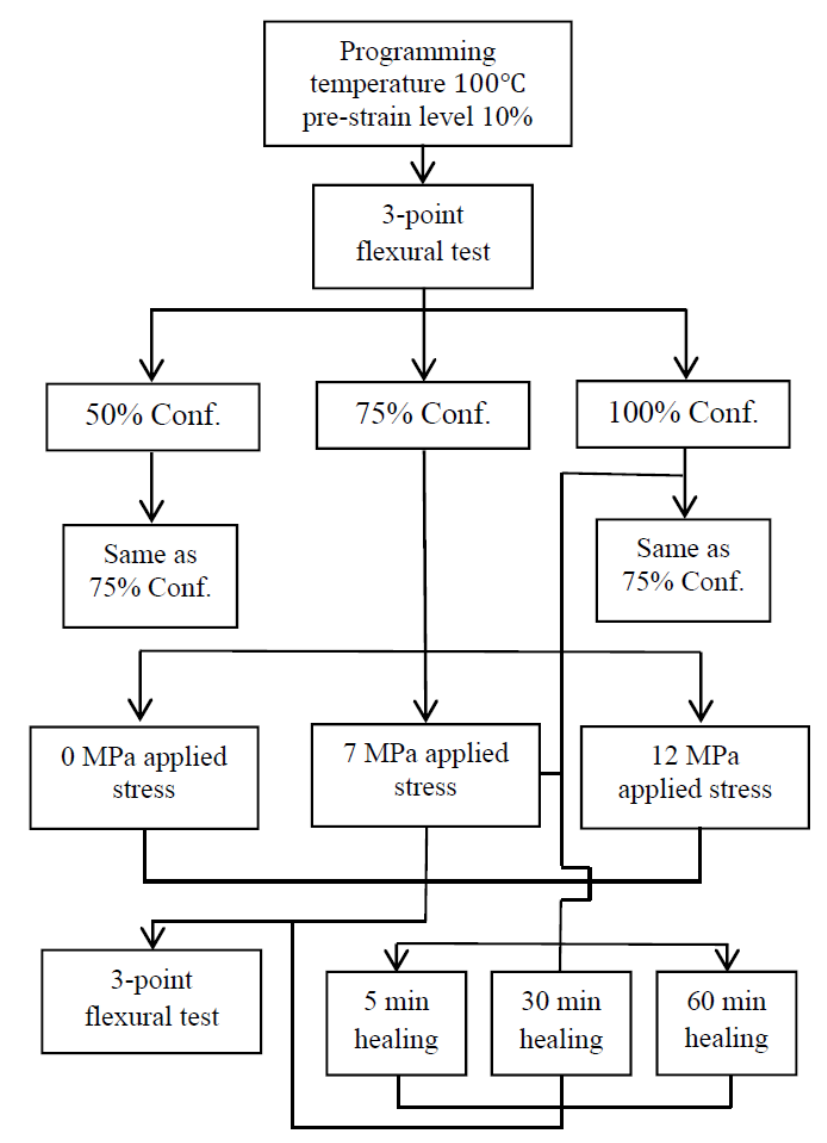

Figure 4. Experimental procedure flowchart 


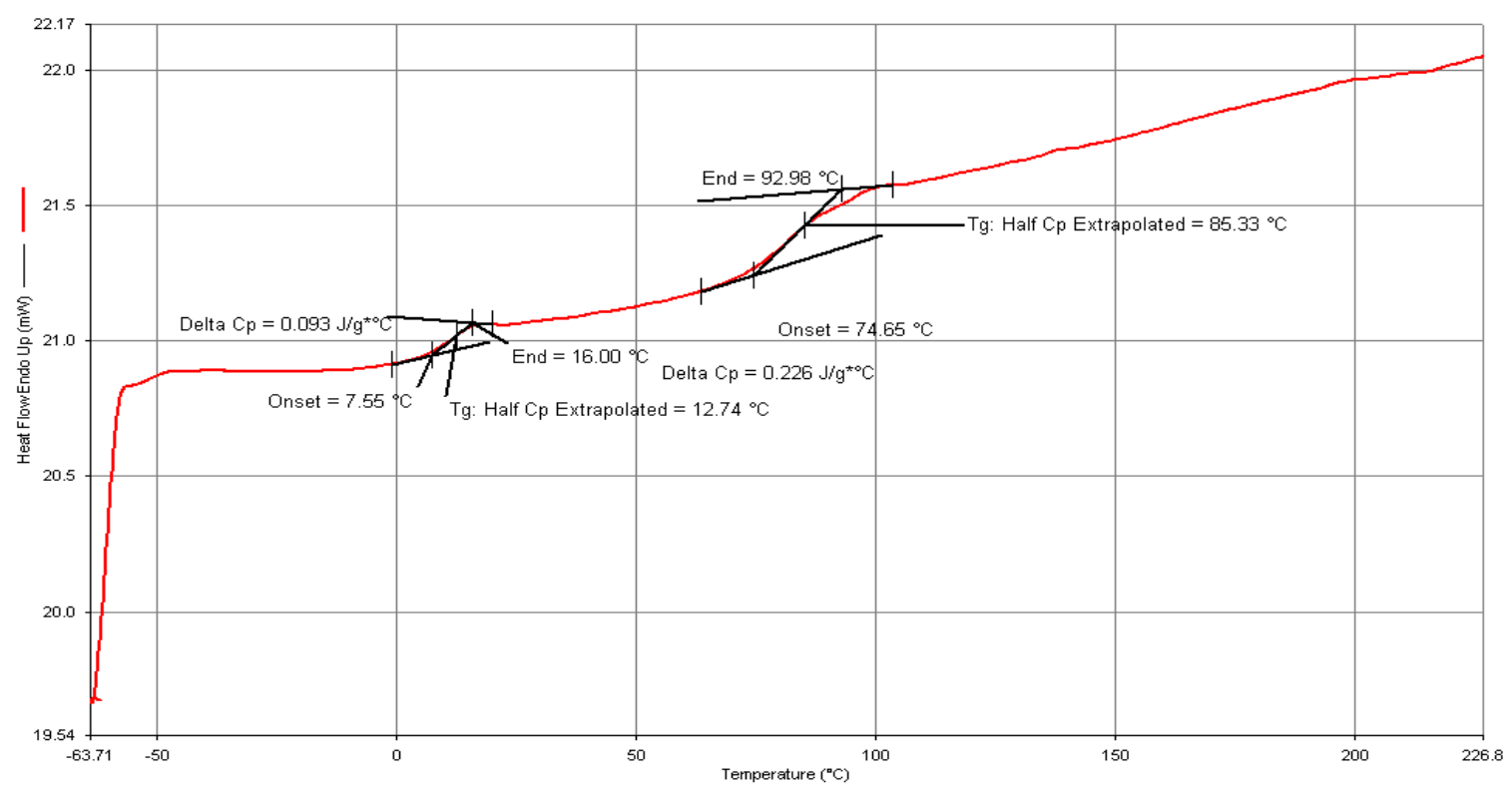

Figure 5. Results of DSC for CP-PSMP material

\subsection{Thermomechanical programming}

Thermomechanical programming was performed as per the scheme discussed in section 2.5. Figure 6 shows the stress-strain-time plot for a typical thermomechanical programming cycle of a CP-PSMP specimen compressively strained to $10 \%$. It should be noted that the temperature was held at a constant $100{ }^{\circ} \mathrm{C}$ during the pre-straining of the specimens. 


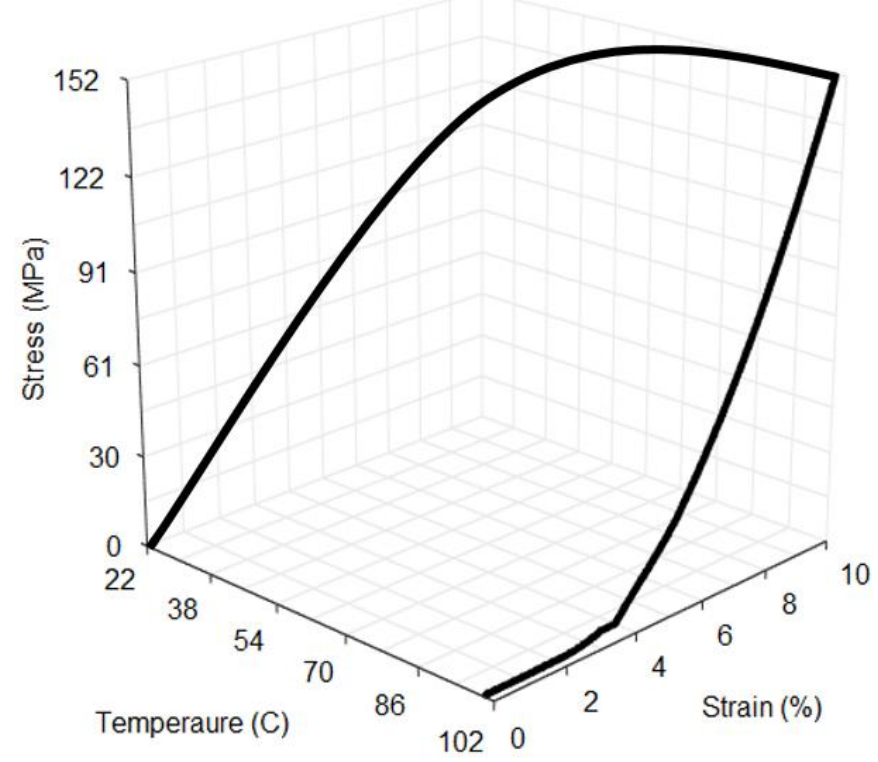

Figure 6. Typical Stress-Strain-Temperature plot representative of what would be seen for specimens pre-strained to $10 \%$ at $100^{\circ} \mathrm{C}$.

\subsection{Three-point flexural test results}

As discussed, three point flexural tests were performed both before and after healing in order to quantify healing efficiency via strength recovery (Equation 1). Figure 7 shows a typical force-deflection plot for a specimen post programming and post healing.

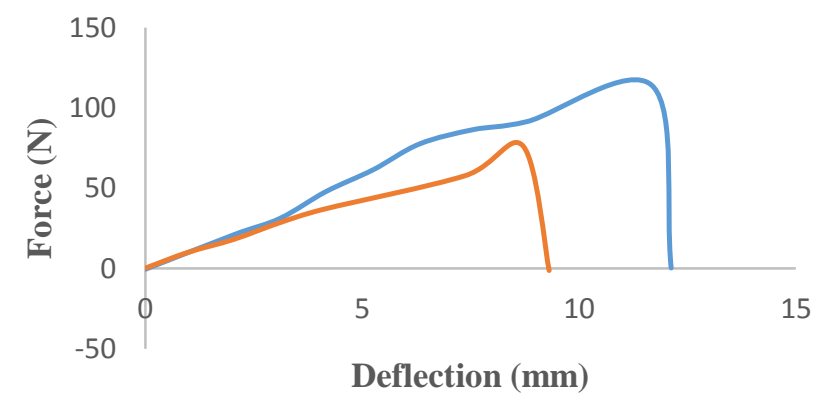

- Post Programming Post Healing

Figure 7. Flexural strength before and after healing for a specimen healed under $75 \%$ confinement and $7 \mathrm{MPa}$ axial stress. 
Although the results shown in Figure 7 are for a specimen healed under $75 \%$ confinement with $7 \mathrm{MPa}$ applied axial constraint stress, the trend seen is typical for all specimens healed, regardless of their healing method. This trend has a two-fold implication: (1) the peak bending load decreases after it is healed as compared to the specimen without damage, and (2) the ductility decreases and the specimen becomes more brittle after healing. In specimens with higher healing efficiencies, the two plots in Figure 7 become closer to each other, i.e. the post healing plot approaches the post programming plot as the healing efficiency approaches $100 \%$. Thus, through effective healing means, the specimen can regain its pre-damaged mechanical properties.

\subsection{Effect of confinement on healing efficiency}

As hypothesized, varying the level of confinement during healing proved to influence the strength recovery of the CP-PSMP specimens. In each case, the healing efficiency showed a trend of increasing with increasing confinement level. Figure 8 shows a typical trend for the strength recovery increase with increasing confinement for specimens healed at $0 \mathrm{MPa}$ applied axial stress (i.e., allow free shape recovery in the axial direction during the healing process). To further investigate healing under axial constraint, specimens were healed at varying levels of applied axial compressive stress in order to simulate varying recovery stress levels (to be discussed in section 3.5). This was done by exerting a compressive stress on the ends of the specimens in the axial direction, during healing via the QTEST/150 MTS machine. As can be

seen in Figures 9 and 10, the overall healing efficiency of the specimens tested at 0, 7, and 12 MPa applied axial stress increase as the axial constraint level increases. The maximum average healing efficiency (for specimens healed for 30 minutes) of $91.02 \%$ was attained through healing under $100 \%$ radial confinement at an applied axial stress of $12 \mathrm{MPa}$. 


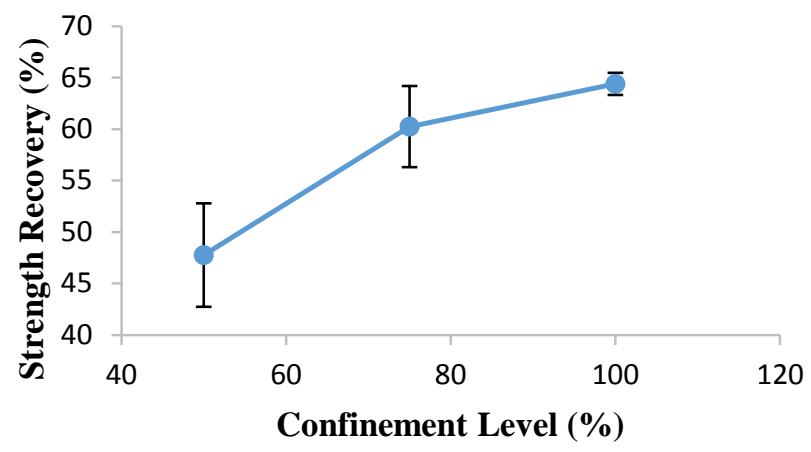

Figure 8. Healing efficiency versus radial confinement level for specimens healed under zero axial constraint.

\subsection{Effect of confinement on recovery stress}

Recovery stress, defined as the stress the specimen exhibits during recovery (or attempt at recovery) to its original shape, was also investigated. To do this, specimens were healed at 50, 75, and 100\% lateral confinement with no external axial load applied. To that end, after the specimen was placed in the correspondingly sized tube, the QTEST/150 MTS machine cross heads were brought into contact with the two ends of the specimen. The MTS machine was then set in two-speed tensile mode with the speed set to zero in order to record the recovery stress as the specimens attempted to regain their original shape during recovery. Next the specimen was heated to the healing temperature of $135^{\circ} \mathrm{C}$ and allowed to recover for 30 minutes after that uniform temperature was reached. 


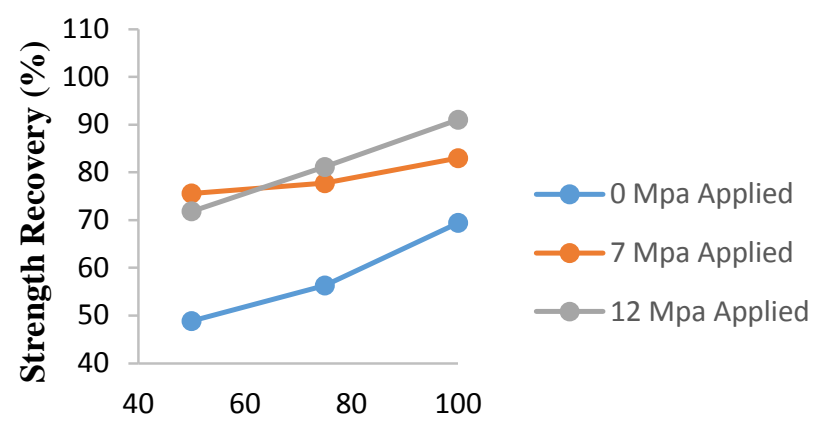

Confinement Level (\%)

Figure 9. Healing efficiency versus confinement level for specimens healed at 0,7 , and $12 \mathrm{MPa}$ applied axial constraint stress. Note to preserve graph clarity error bars were omitted and the average is shown for each data point (errors can be found in Table 1).

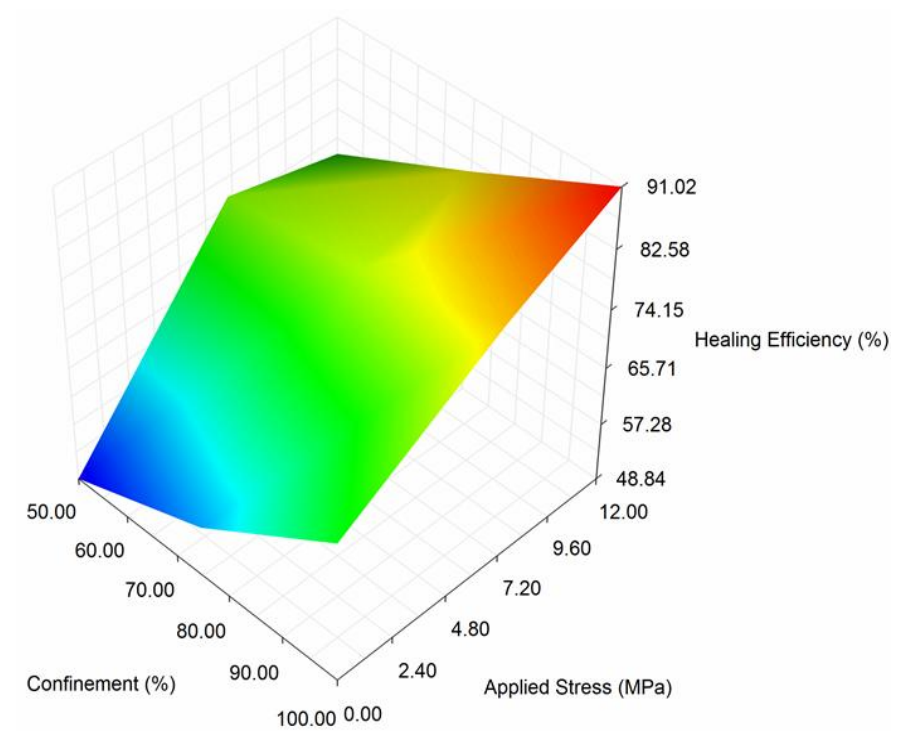

Figure 10. Surface graph showing the variation of healing efficiency with radial confinement level and applied axial constraint stress.

It was found that as the lateral confinement level increased, so did the recovery stress (see Figure 11). Moreover, Figure 8 suggests that the higher the level of lateral confinement, the higher the healing efficiency. Therefore, there exists a direct correlation between the level of radial confinement and the healing efficiency; namely, the healing efficiency increases with increasing radial confinement. The reason for this is twofold. First, as the level of lateral 
confinement (and thus the length of the specimen thermally activated) is increased, more of the total polymer chains within the specimen are thermally activated. More polymer chain activation leads to higher recovery stress because more of the total polymer chains in the specimen are trying to recover to their original shape. This translates to more force is created to close the crack and thus leads to higher strength recovery. This is apparent in Figure 8 where there was no external axial stress applied. The only stress involved in structurally closing the internal crack was the axial recovery stress produced by the specimen itself. Secondly, higher recovery stress can also contribute to better healing through pressure assisted penetration or diffusion of the liquefied thermoplastic particles during healing [39]. Therefore, the higher recovery stress produces a synergistic couple that is very conducive to crack closure and penetration of the $\mathrm{CP}$ molecules into the fractured PSMP matrix. Indeed, this increase in axial recovery stress with increasing lateral confinement is one major reason that better healing efficiencies are obtained with higher levels of lateral confinement.

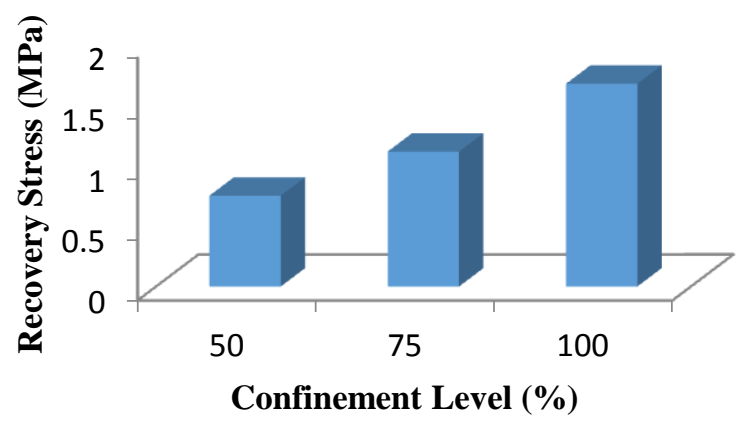

Figure 11. Recovery stress variation with radial confinement level.

\subsection{Healing time influence on healing efficiency}

In this study, three healing times were investigated; 5, 30, and 60 minutes. It was found that there exists a positive correlation between the healing time and the healing efficiency over 
the range of values studied; see Figure 12. For this portion of the study the lateral confinement level and applied axial constraint stress were held constant at $100 \%$ and $12 \mathrm{MPa}$, respectively. There was quite a difference in the healing efficiency seen over the range of healing times studied with the minimum efficiency being $79.28 \%$ at 5 minutes and a maximum of $97.59 \%$ at 60 minutes. This may be due to more time allowed for the PSMP to close the cracks via the shape memory effect and more time for thermoplastic molecule diffusion. It may also be due to the time required for the heat transfer from the heating blanket wrapped around the outside of the confining tube to the specimen inside the tube.

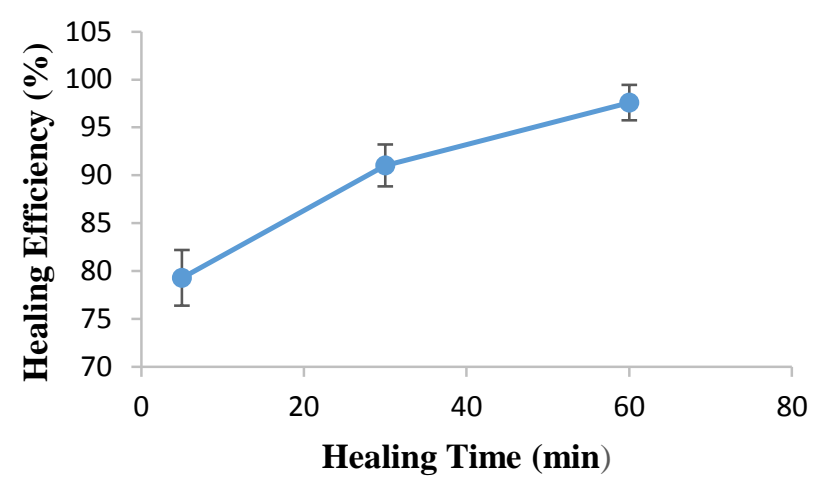

Figure 12. Healing efficiency versus healing time.

It is expected that longer periods of healing (longer than 60 minutes) would not necessarily produce higher healing efficiencies. Indeed, this was seen in specimens healed for approximately 2 hours during a preliminary study of the healing time. The healing efficiency of these specimens was in the vicinity of that of specimens healed for 60 minutes. An in depth study into healing times above 60 minutes was not extensively investigated, however, because it was out of the scope of this study. Further investigation into the healing time should be the subject of future research. 
Table 1 provides a master data list for each of the experiments discussed thus far (as listed in the flowchart in Figure 4).

Table 1. List of all relevant experimental results.

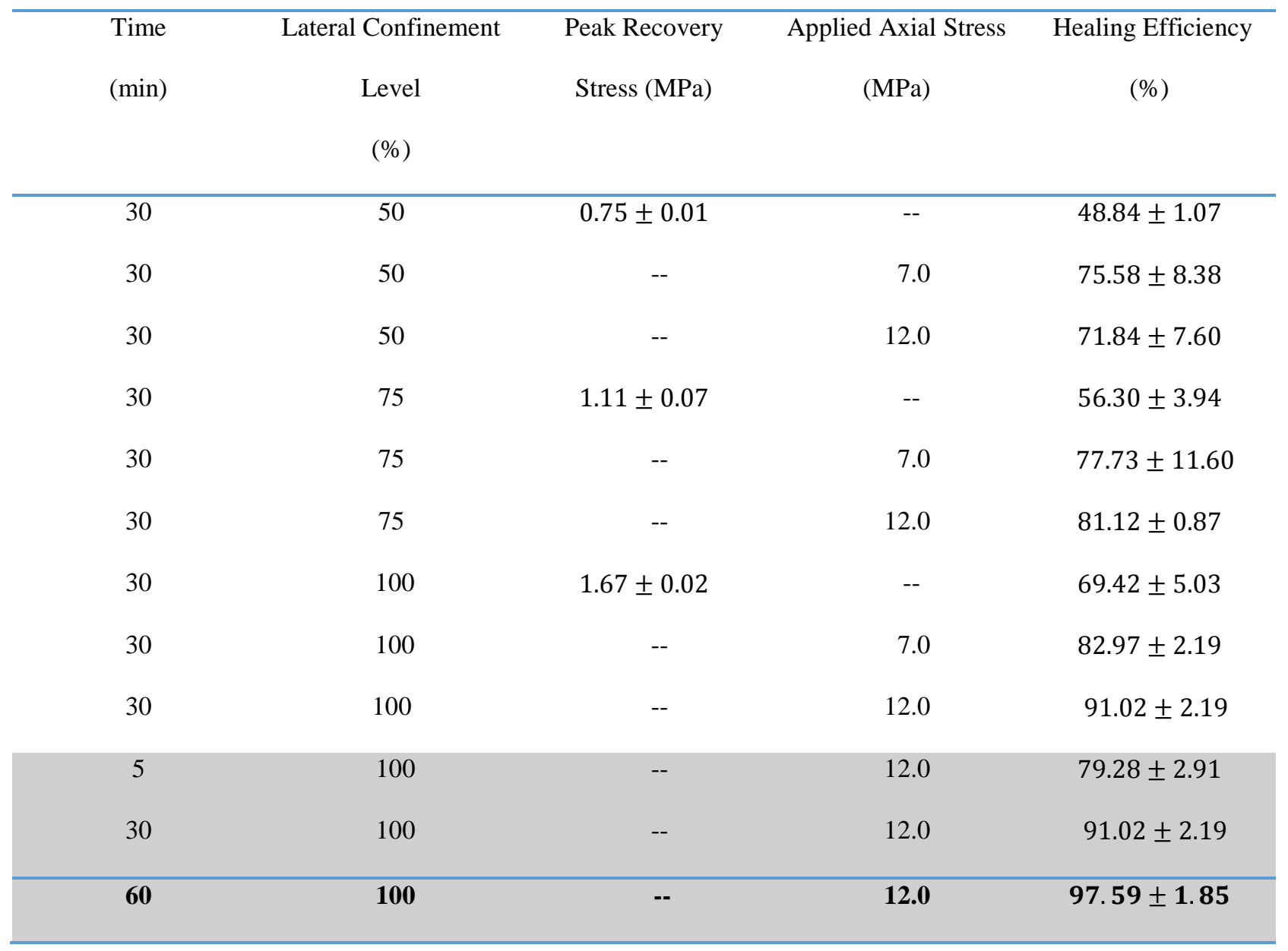

\subsection{Effect of programming prestrain level on recovery stress}

In this study, the applied axial constraint stress levels were varied (0, 7, and $12 \mathrm{MPa})$ to simulate varying levels of axial constraints. In order to investigate if the composite itself can have such level of recovery stress or not, two additional prestrain levels were used to 
compressively programm the composite cylinders, which were $16 \%$ and $19 \%$, respectively. As found by Li and Uppu [32], compressive prestrain level higher than $17 \%$ will damage the composite under lateral confinement. Therefore, the upper limit was chosen as $19 \%$. Specimens pre-strained to $16 \%$ produced recovery stress levels of approximately $11 \mathrm{MPa}$ when recovered under $100 \%$ lateral confinement. However, further increasing the pre-strain level to $19 \%$ proved to decrease the recovery stress to around $2 \mathrm{MPa}$. This is probably due to the high strain level caused damage to the composite. Similar observations were also made by Li and Uppu [32]. Thus, using pre-strain levels of around $16 \%$ can indeed provide recovery stress levels close to the maximum (12 MPa) used in this study. It is found that, cold programming, i.e., programming in the glass state, may increase the recovery stress of either thermosetting SMPs or thermoplastic SMPs [40-44].

\subsection{SEM observation}

To further validate the results of this study, images were taking of the CP-PSMP fracture surface using a scanning electron microscope. As previously discussed, in order for effective healing of cracks and damage, the cracks must first be effectively closed. Once the cracks have been closed through confined recovery at a temperature above the $T_{g}$ of the PSMP matrix, further heating will cause melting of the thermoplastic healing agents which will then be sucked into the narrowed cracks by capillary forces. However, insufficient axial constraint stress leads to the presence of unclosed cracks within the material during healing, which ultimately leads to limited healing efficiency. Figure 13 shows a typical healed crack seen on the surface of the CP-PSMP specimen after healing under $50 \%$ lateral confinement at $0 \mathrm{MPa}$ externally applied axial constraint stress, i.e., the axial constraint stress is by the recovery stress only (about $0.75 \mathrm{MPa}$ ). As can be seen in the figure, the crack is not completely closed, suggesting that the recovery 
stress of the composite healed under 50\% lateral confinement $(\sim 0.75 \mathrm{MPa})$ is not enough to fully close cracks during healing. Inspection of image Figure 13 (b) further validates this claim. In this image, it is seen that the thermoplastic particles have melted into the crack; however, it seems that the crack may have not been closed enough to effectively "glue" the two cracked surfaces together by the thermoplastics. In contrast, Figure 14 shows a fracture surface healed under $100 \%$ lateral confinement with $7 \mathrm{MPa}$ axial constraint stress. In Figure 14(a), it can be seen that the healed crack is completely closed suggesting that full lateral confinement coupled with a relatively high axial constrain stress of $7 \mathrm{MPa}$ produces effective closure of cracks within the specimen. Further magnification of the region seen in image Figure 14 (b) validates this claim. As shown in Figure 14 (b), the crack has been completely closed with only the outline remaining as evidence of its existence. Therefore, the recovery stress by the $10 \%$ compressive programming is not sufficient to close the cracks. External axial compressive load or higher programming strain (such as $16 \%$ prestrain as discussed in section 3.7) is needed to close the crack and to increase the healing efficiency.

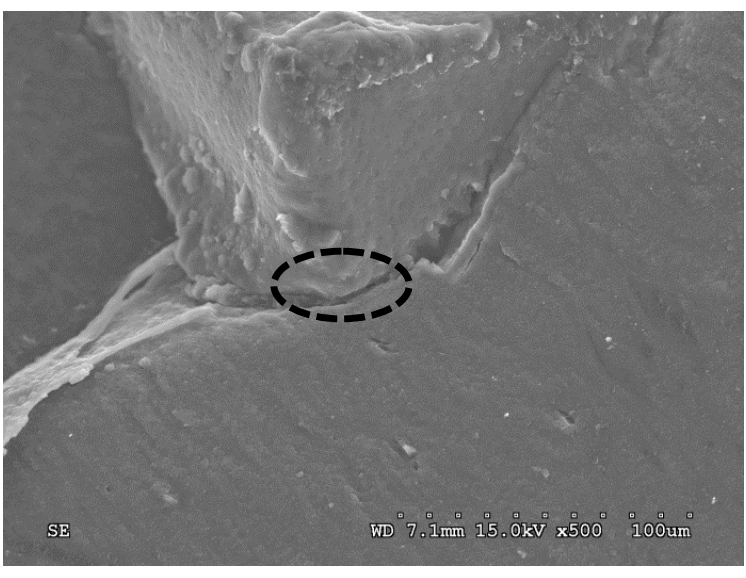

(a)

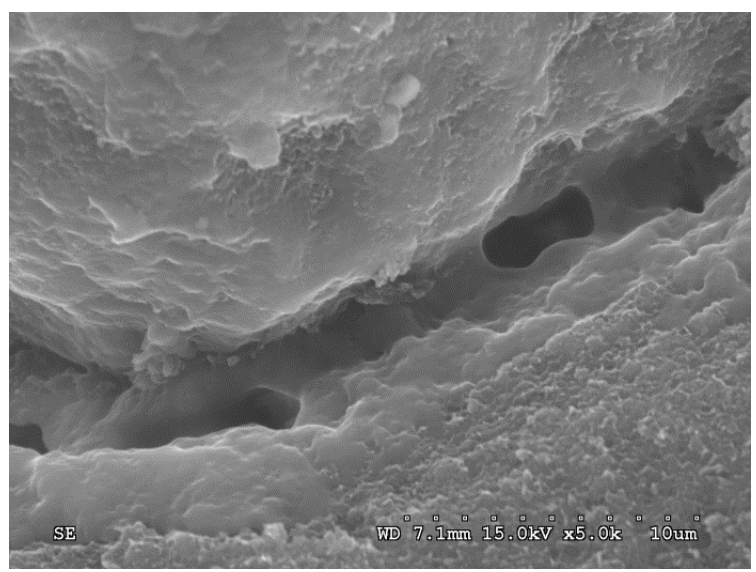

(b)

Figure 13. Crack seen on the fracture surface of CP-PSMP specimen healed under 50\% lateral confinement with zero externally applied axial load. (a) shows the crack under consideration and (b) shows the highlighted region of the crack at higher magnification. 

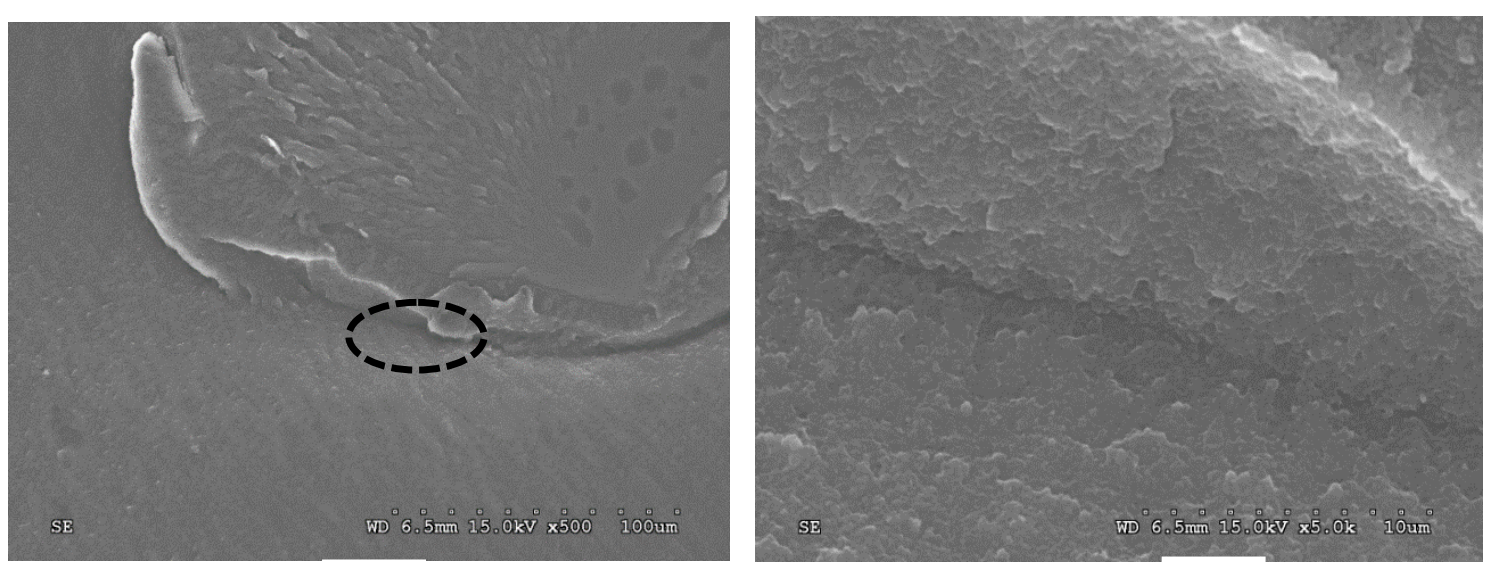

Figure 14. Effectively closed crack seen on the fracture surface of CP-PSMP specimen healed under 100\% lateral confinement at $7 \mathrm{MPa}$ of axial constraint stress. (a) Shows the sealed crack and (b) shows the highlighted region in (a) under higher magnification.

\section{Conclusion}

Self-healing shape memory polymer composites are an exciting field of study and much research has been conducted in this area thus far. In the present study it is established that almost complete strength recovery is possible by local heating, and with appropriate constraint levels and heating times. Based on the systematic experimental program, the following conclusions are established:

- Higher lateral confinement, stronger axial constraint, and longer healing time are all positive factors in increasing the healing efficiency, at least within the range used in this study.

- The lateral confinement level and axial stress recovery are coupled. Higher lateral confinement level leads to higher recovery stress in the axial direction.

- Increasing the compression programming prestrain level increases the axial recovery stress. With a prestrain level of $16 \%$, the recovery stress is about $11 \mathrm{MPa}$, which is similar to the maximum applied axial constraint used in this study (12MPa). Therefore, it is expected that, using the axial recovery stress alone, the composite may have a similar 
healing efficiency without externally applying an axial load, if the programming prestrain is about $16 \%$.

- $\quad$ Further increasing the programming prestrain level above $16 \%$ does not necessarily increase the recovery force. It may cause damage to the composite.

\section{Acknowledgement}

This study was financially supported by National Science Foundation under grant number CMMI 1333997, and Army Research Office under grant number W911NF-13-1-0145.

\section{References}

[1] Bekas DG, Tsirka K, Baltzis D, Paipetis AS. Self-healing materials: A review of advances in materials, evaluation, characterization and monitoring techniques. Composites Part B: Engineering, 87: 92-119, (2016).

[2] Li G. Self-Healing Composites: Shape Memory Polymer Based Structures. ISBN-13: 978-1118452424, ISBN-10: 1118452429, John Wiley \& Sons, Inc., West Sussex, UK, (2014).

[3] Chen X, Dam MA, Ono K, Mal A, Shen H, Nutt SR, Sheran K, Wudl F. A thermally remendable cross-linked polymeric material. Science, 295:1698-1702, (2002).

[4] Varley R, van der Zwaag S. Towards an understanding of thermally activated self-healing of an ionomer system during ballistic penetration. Acta Materialia, 56:5737-5750, (2008).

[5] Sijbesma RP, Beijer FH, Brunsveld L, Folmer BJB, Hirschberg JHKK, Lange RFM, Lowe JKL, Meijer EW. Reversible polymers formed from self-complementary monomers using quadruple hydrogen bonding. Science, 278:1601-1604, (1997). 
[6] Hemmelgarn CD, Margraf TW, Havens DE, Reed, JR. JL, Snyder LW, Louderbaugh A, Dietsch BA. Composites Self-healing System. US PCT No. PCT/US08/60055, (2008).

[7] Montarnal, D, Capelot M, Tournilhac F, Leibler L. Silica-like malleable materials from permanent organic networks. Science, 334:965-968, (2011).

[8] Dry CM. Alteration of matrix permeability and associated pore and crack structure by timed release of internal chemicals. Ceramic Transactions, 16:729-768, (1991).

[9] Bleay SM, Loader CB, Hawyes VJ, Humberstone L, Curtis PT. A smart repair system for polymer matrix composites. Composites Part A-Applied Science and Manufacturing, 32:1767-1776, (2001).

[10] Es-haghi H, Mirabedini SM, Imani M, Farnood RR. Mechanical and self-healing properties of a water-based acrylic latex containing linseed oil filled microcapsules: Effect of pre-silanization of microcapsules' shell compound, Composites Part B: Engineering, 85: 305-314.

[11] Li Q, Siddaramaiah, Kim NH, Hui D, Lee JH. Effects of dual component microcapsules of resin and curing agent on the self-healing efficiency of epoxy. Composites Part B: Engineering, 55: 79-85, (2013).

[12] Li Q, Mishra AK, Kim NH, Kuila T, Lau KT, Lee JH. Effects of processing conditions of poly(methylmethacrylate) encapsulated liquid curing agent on the properties of selfhealing composites. Composites Part B: Engineering, 49: 6-15, (2013).

[13] Guadagno L, Longo P, Raimondo M, Naddeo C, Mariconda A, Vittoria V, Iannuzzo G, Russo S. Use of Hoveyda-Grubbs' second generation catalyst in self-healing epoxy mixtures. Composites Part B: Engineering, 42: 296-301, (2011). 
[14] Zako M, Takano N. Intelligent material systems using epoxy particles to repair microcracks and delamination damage in GFRP. Journal of Intelligent Material Systems and Structures, 10:836-841, (1999).

[15] Hayes SA, Jones FR, Marshiya K, Zhang W. A self-healing thermosetting composite material. Composites Part A-Applied Science and Manufacturing, 38:1116-1120, (2007).

[16] Nji J, Li G. Damage healing ability of a shape memory polymer based particulate composite with small thermoplastic contents. Smart Materials and Structures, 21: paper number 025011 (10 pages), (2012).

[17] Nji J, Li G. A biomimic shape memory polymer based self-healing particulate composite. Polymer, 51:6021-6029, (2010).

[18] White SR, Sottos NR, Geubelle PH, Moore JS, Kessler MR, Sriram SR, Brown EN, and Viswanathan S. Autonomic healing of polymer. Nature, 409:794-797, (2001).

[19] Lee J, Zhang M, Bhattacharyya D, Yuan YC, Jayaraman K, Mai YW. Micromechanical behavior of self-healing epoxy and hardener-loaded microcapsules by nanoindentation. Materials Letters, 76:62-65, (2012).

[20] Pang JWC, Bond IP. A hollow fibre reinforced polymer composite encompassing selfhealing and enhanced damage visibility. Composites Science and Technology, 65:17911799, (2005).

[21] Trask RS, Williams GJ, Bond IP. Bioinspired self-healing of advanced composite structures using hollow glass fibres. Journal of the Royal Society Interface, 4:363-371, (2007).

[22] Escobar MM, Vago S, Vázquez A. Self-healing mortars based on hollow glass tubes and epoxy-amine systems. Composites Part B: Engineering, 55: 203-207, (2013). 
[23] Toohey KS, Sottos NR, Lewis JA, Moore JS, White SR. Self-healing materials with microvascular networks. Nature Materials, 6:581-585, (2007).

[24] Williams HR, Trask RS, Knights AC, Williams ER, Bond IP. Biomimetic reliability strategies for self-healing vascular networks in engineering materials. Journal of the Royal Society Interface, 5:735-747, (2008).

[25] Wool RP and O'Connor KM. A theory of crack healing in polymers. Journal of Applied Physics, 52: 5953-5963, (1981).

[26] Wool RP. Self-healing materials: a review. Soft Matter, 4: 400-418, (2008).

[27] Self-healing Polymers - From Principles to Applications. Binder WH, ed., Wiley-VCH, ISBN 978-3-527-33439-1, (2013).

[28] Li G and John M. A smart syntactic foam under multiple impact. Composites Science and Technology 68: 3337-3343, (2008).

[29] John M and Li G. Self-Healing of Sandwich Structures with Grid Stiffened Shape Memory Polymer Syntactic Foam Core. Smart Materials and Structure, 19: paper number 075013 (12 pages), (2010).

[30] Nji J and Li G. A Self-Healing 3D Woven Fabric Reinforced Shape Memory Polymer Composite for Impact Mitigation. Smart Materials and Structures, 19: paper number 035007 (9 pages), (2010).

[31] Li G and Nettles D. Thermomechanical characterization of a shape memory polymer based self-repairing syntactic foam. Polymer, 51: 755-762, (2010).

[32] Li G and Uppu N. Shape memory polymer based self-healing syntactic foam: 3-D confined thermomechanical characterization. Composite Science and Technology. 70:1419-1427, (2010). 
[33] Wei H, Yao Y, Liu Y, Leng L. A dual-functional polymeric system combining shape memory with self-healing properties. Composites Part B: Engineering, 83: 7-13, (2015).

[34] Li G, Meng H, and Hu J. Healable Thermoset Polymer Composite Embedded with Stimuli-responsive Fibers. Journal of the Royal Society Interface, 9: 3279-3287, (2012).

[35] Neuser S, Michaud V, White SR. Improving solvent-based self-healing materials through shape memory alloys. Polymer, 53: 370-378, (2012).

[36] Zhang P and Li G. Healing-on-demand Composites Based on Polymer Artificial Muscle. Polymer, 64: 29-38, (2015).

[37] Lendlein A and Kelch S. Shape-Memory Polymers. Angewandte Chemie International Edition 41:2035-2057, (2002).

[38] Yougoubare YQ and Pang SS. Effects of programming and healing temperatures on the healing efficiency of a confined healable polymer composite. Smart Materials and Structures, 23: paper number 025027 (9 pages), (2014).

[39] Shojaei A, Sharafi S, and Li G. A Multiscale Theory of Self-Crack-Healing with Solid Healing Agent Assisted by Shape Memory Effect. Mechanics of Materials, 81: 25-40, (2015).

[40] Yang Q and Li G. Investigation into stress recovery behavior of shape memory polyurethane fiber. Journal of Polymer Science-Part B: Polymer Physics, 52: 1429-1440 (2014).

[41] Li G and Zhang P. A Self-healing Particulate Composite Reinforced with Strain Hardened Short Shape Memory Polymer Fibers. Polymer, 54:5075-5086, (2013). 
[42] Li G, Ajisafe O, Meng H. Effect of Strain Hardening of Shape Memory Polymer Fibers on Healing Efficiency of Thermosetting Polymer Composites. Polymer, 54:920-928, (2013).

[43] Li G and Shojaei A. A Viscoplastic Theory of Shape Memory Polymer Fibers with Application to Self-Healing Materials. Proceedings of the Royal Society A-Mathematical Physical and Engineering Sciences, 468: 2319-2346, (2012).

[44] Li G and Xu W. Thermomechanical Behavior of Thermoset Shape Memory Polymer Programmed by Cold-Compression: Testing and Constitutive Modeling. Journal of the Mechanics and Physics of Solids, 59:1231-1250, (2011). 